\title{
Some statements for bi-pseudo-integrals and the role on reconstruction of the pseudo-additive measures
}

\author{
Dhurata Valera \\ Aleksandër Xhuvani University, NSF, Mathematics Department, Elbasan, ALBANIA \\ E-mail: dhurata_valera@hotmail.com
}

Copyright $\odot 2015$ Dhurata Valera. This is an open access article distributed under the Creative Commons Attribution License, which permits unrestricted use, distribution, and reproduction in any medium, provided the original work is properly cited.

\begin{abstract}
With the support of some very important and special generators, are given some details about the properties of bipseudo-integrals and above all, for the first bi-pseudo-integral the relations with integral Lebesgue are listed. Further, will be shown pseudo-linearity of bi-pseudo-integrals and some investigations in reconstructions of pseudo-additive measures by bi-pseudo-integrals synthesized the reciprocal relationship between pseudo-additive measure and bipseudo-integral.
\end{abstract}

Keywords: Bi-Pseudo-Integral; Pseudo-Additive Measure; Generator, Pseudo-Operations; Reconstruction.

\section{Introduction}

In section 2 are presented some of the most important generators $\bar{g}$ that play an important role in definitions of bipseudo-integrals and their properties. There are shown the connections between the $\left(\oplus_{g_{a, r}} \odot_{g_{a, r}}\right)-$ integral and the Lebesgue integral of non-negative function so, in the case of $\left(\bigoplus_{g_{1, r}}, \bigodot_{g_{1, r}}\right)=\left(\oplus_{g_{1, r}}\right.$, ' $)$, the Marinová's integral (as the first bi-pseudo-integral) is the Lebesgue integral [3], [7], [11]. In the case of $(\bigoplus=\vee=\max )$, the Marinová's integral $\left(M-\int_{X}^{(V, \odot)}\right)$ leads to the Shilkret's integral [12]. For $(\bigoplus \neq V=\max )$ is discovered the connection between the $\left(\mathrm{M}-\int_{\mathrm{X}}\left(\oplus_{g_{a, r}} \odot_{g_{a, r}}\right)\right)$ and the Lebesgue integral, but Kolesárová in [7] has explained the reasons for another definition of the $\left(\bar{\oplus}_{\bar{g}_{a, r}} \bar{\odot}_{\bar{g}_{a, r}}\right)$ - integral of real functions which is more appropriate than the definition that was given in [11]. The generalization of Bi-Pseudo-Integral and relations with Lebesgue integral are treated in section 3. Different properties of integrals with respect to $\bar{\oplus}_{\bar{g}}$ - measure and $\bar{V}$-measure for $\mathrm{f}$ - RMF are caused by the essential difference between $\bar{\bigoplus}_{g_{a, r}}$ and $\bar{V}$ [3], [7], [11], [12]. The notion of modified pseudo-additive measure $\left(\bar{\oplus}-m_{\bar{g}}\right)$ by $\bar{g}$ - transform is followed and completed with the meaning of bi-pseudo-integral of modified function $\left(f_{\bar{g}}\right)$ with respect to a $m_{\bar{g}}$ in [10]. More links between different types of the bi-pseudo-integrals are noted in [10]. For the reconstruction of pseudo-additive measures some statements are treated in section 4. Further, the results are summarized in section 5 .

\section{Preliminary notes}

Let a generator $\bar{g}:[-\infty,+\infty] \rightarrow[-\infty,+\infty]$ be a continuous, monotone strictly increaing unbouded function of the pseudo-addition $\bar{\oplus}$ on the interval $[-\infty,+\infty]$, such that $\bar{g}(0)=0_{\oplus}, \bar{g}(1)=1_{\odot}, \bar{g}(+\infty)=+\infty$, with the convention $0 \cdot(+\infty)=0$ and some valued undefined (or an odd extension of a given generator $g$ from $[0,+\infty]$ to $[-\infty,+\infty]$, briefly $\bar{g}(\mathrm{x})=\operatorname{sgn} \mathrm{x} \cdot g(|\mathrm{x}|), \mathrm{x} \in[-\infty, \infty])$. The concept of pseudo-arithmetical operations $\{\oplus, \odot, \ominus, \oslash\}$ first was introduced on $[0,+\infty]$ interval and then to the whole extended real line $[-\infty,+\infty]$ [1], [3], [4], [5], [6], [7], [14], [16]. 
Following Mesiar and Rybá rik [4], the binary operations $(\bar{\oplus}, \bar{\odot})$ (pseudo-addition, pseudo-multiplication) are respectively the binary function that [3], [7], [11] fulfill the system of axioms $(\bar{\oplus}-\mathrm{A} .1 \div \mathrm{A} .7)$ and $(\bar{\odot}-\mathrm{A} .1 \div \mathrm{A} .7)$. The system of the axioms $(\bigoplus, \odot$-A.1 $\div$ A. 5) was formulated by the system of the Axioms of Sugeno and Murofushi [8], [15], [16]. Then, the sistem of pseudo-arithmetical operations $\{\bar{\oplus}, \bar{\odot}, \bar{\ominus}, \bar{\oslash}\}=\left\{\bar{\oplus}_{\bar{g}}, \bar{\bigodot}_{\bar{g}}, \bar{\ominus}_{\bar{g}}, \bar{\oslash}_{\bar{g}}\right\}$ generated by this function $\bar{g}$, is said to be a consistent sistem [3], [7], [11].

So, for $\mathrm{x}, \mathrm{y} \in[-\infty,+\infty]$, let $\bar{g}$ be a generator on $[-\infty,+\infty]$, perhaps with some valued undefined [4], [8], [11], [13]. There are selected some most important generators $\bar{g}$ and $\bar{g}-$ calculus derived from them [2], [4], [5], [11], [13] are listed in the Table 1.

\begin{tabular}{|c|c|c|}
\hline$\overline{\boldsymbol{g}}(\boldsymbol{x})=\overline{\boldsymbol{g}}_{a, r}(\boldsymbol{x})$ & $\left\{\bar{\oplus}_{\bar{g}}, \bar{\bigodot}_{\bar{g}}, \bar{\ominus}_{\bar{g}}, \bar{\oslash}_{\bar{g}}\right\}$ & Conditions $(\boldsymbol{a}, \boldsymbol{r}, \boldsymbol{x}, \boldsymbol{y})$ \\
\hline $\bar{g}_{1,1}(x)=x$ & $\begin{array}{l}x \bar{\oplus}_{\bar{g}_{1,1}} y=x+y \\
x \bar{\bigodot}_{\bar{g}_{1,1}} y=x \cdot y \\
x \bar{\Theta}_{\bar{g}_{1,1}} y=x-y \\
x \bar{\oslash}_{\bar{g}_{1,1}} y=x / y\end{array}$ & $r=1, a=1$ \\
\hline $\bar{g}_{a, 1}(x)=a \cdot x$ & $\begin{array}{l}x \bar{\oplus}_{\bar{g}_{a, 1}} y=x+y \\
x \bar{\odot}_{\bar{g}_{a, 1}} y=a \cdot(x \cdot y) \\
x \bar{\ominus}_{\bar{g}_{a, 1}} y=x-y \\
x \bar{\emptyset}_{\bar{g}_{a, 1}} y=a^{-1} \cdot(x / y)\end{array}$ & $r=1, a>0$ \\
\hline $\bar{g}_{1, r}(x)=x^{r}$ & $\begin{array}{l}x \bar{\oplus}_{\bar{g}_{1, r}} y=\left(x^{r}+y^{r}\right)^{1 / r} \\
x \bar{\bigodot}_{\bar{g}_{1, r}} y=x \cdot y \\
x \bar{\ominus}_{\bar{g}_{1, r}} y=\left(x^{r}-y^{r}\right)^{1 / r} \\
x \bar{\oslash}_{\bar{g}_{1, r}} y=x / y\end{array}$ & $r \geq 1, a=1$ \\
\hline$\overline{\boldsymbol{g}}_{\boldsymbol{a}, \boldsymbol{r}}(\boldsymbol{x})=\boldsymbol{a} \cdot \boldsymbol{x}^{r}$ & $\begin{array}{l}x \bar{\oplus}_{\bar{g}_{a, r}} y=\left(x^{r}+y^{r}\right)^{1 / r} \\
x \bar{\odot}_{\bar{g}_{a, r}} y=a^{1 / r} \cdot(x \cdot y) \\
x \bar{\ominus}_{\bar{g}_{a, r}} y=\left(x^{r}-y^{r}\right)^{1 / r} \\
x \bar{\oslash}_{\bar{g}_{a, r}} y=a^{-1 / r} \cdot(x / y)\end{array}$ & $r \geq 1, a>0$ \\
\hline
\end{tabular}

If $a=1$, we have the follow form of the generator i.e., the normed generator $\bar{g}(x)=\bar{g}_{1, r}(x)=x^{r}$ and $\bar{g}(1)=$ $\bar{g}_{1, r}(1)=1$. Also, easily can control that $\bar{g}_{a, r}{ }^{-1}(a)=1, \bar{g}_{a, 1}{ }^{-1}(a)=1$ and $\bar{g}_{a, r}{ }^{-1}(1)=1, \bar{g}_{a, 1}{ }^{-1}(1)=1$ [3], [4], [7], [11], [13].

Definition 2.1.1: For a simple non-negative measurable function (in short form $s$ - SNNMF) defined on X, $s(x)=\sum_{i=1}^{n} \propto_{i} \cdot \mathbb{I}_{A_{i}}$ where sets $A_{i} \in \mathcal{A}, A_{i} \neq A_{j}, A_{i} \cap A_{j}=\emptyset$, for $i \neq j, i, j=\overline{1, n}, 0<\propto_{i}<+\infty$, then

$\left(M-\int_{X}^{\left(\oplus_{g_{a, r}}, \odot_{g_{a, r}}\right)}\right)(m, s-S N N M F)=\int_{X}^{\left(\oplus_{g_{a, r}} \odot_{g_{a, r}}\right)} s \bigodot_{g_{a, r}} d m=\bigoplus_{i=1}^{\infty} \propto_{i} \bigodot_{g_{a, r}} m\left(A_{i}\right)$ [3], [7], [11].

Definition 2.1.2: For a non-negative measurable function $(f-N N M F), f: X \rightarrow[0,+\infty]$, then

$$
\begin{aligned}
& \left(M-\int_{X}^{\left(\oplus_{g_{a, r}}, \odot_{g_{a, r}}\right)}\right)(m, f-N N M F)=\int_{X}^{\left(\oplus_{g_{a, r}} \odot_{g_{a, r}}\right)} f \odot_{g_{a, r}} d m= \\
& =\sup \left\{\int_{X}^{\left(\oplus_{g_{a, r}}, \odot_{g_{a, r}}\right)} s \odot_{g_{a, r}} d m: s \leq f, s-\text { is a SNNMF }\right\}
\end{aligned}
$$

And say that the function $\mathrm{f}[3],[7],[11]$ is integrable if

$$
\left(M-\int_{X}^{\left(\oplus_{a, r}, \odot_{g_{a, r}}\right)}\right)(m, f-N N M F)=\int_{X}^{\left(\oplus_{g_{a, r}} \odot_{g_{a, r}}\right)} f \odot_{g_{a, r}} d m<+\infty
$$

Generalized definition by Kolesárová in [7] for $\left(\bar{\oplus}_{\bar{g}}, \bar{\bigodot}_{\bar{g}}\right)$-integral, in case of $f-R M F$ with respect to a $\bar{\bigoplus}_{\bar{g}^{-}}$measure $m$ where $\left.\bar{g}_{a, r}\right|_{[0,+\infty]}=g_{a, r},\left.\bar{\bigoplus}_{\bar{g}_{a, r}}\right|_{[0, \infty]}=\bigoplus_{g_{a, r}}$, is given as bellow.

Definition 2.1.3: [7], [10] For a real measurable function (in short form $\mathrm{f}$ - RMF), $\left(\bar{\oplus}=\bar{\bigoplus}_{\bar{g}} \neq \overline{\mathrm{V}}\right)$, 
$f: X \rightarrow(-\infty,+\infty)$, if at least one of the functions $f^{+}, f^{-}$is integrable, then

$\left(M, K-\int_{X}{ }^{\left(\bar{\oplus}_{\bar{g}}, \bar{\odot}_{\bar{g}}\right)}\right)(m, f-R M F)=\int_{X}^{\left(\bar{\oplus}_{\bar{g}}, \bar{\odot}_{\bar{g}}\right)} f^{+} \bar{\bigodot}_{\bar{g}} d m \bar{\ominus}_{\bar{g}} \int_{X}^{\left(\oplus_{\bar{g}}, \bar{\odot}_{\bar{g}}\right)} f^{-} \bar{\bigodot}_{\bar{g}} d m=$

$=\left(M, K-\int_{X}{ }^{\left(\bar{\oplus}_{\bar{g}}, \bar{\odot}_{\bar{g}}\right)}\right)\left(m, f^{+}\right) \bar{\ominus}_{\bar{g}}\left(M, K-\int_{X}{ }^{\left(\bar{\oplus}_{\bar{g}}, \bar{\odot}_{\bar{g}}\right)}\right)\left(m, f^{-}\right)$.

A function $\mathrm{f}$ is called integrable iff

$-\infty<\left(M, K-\int_{X}{ }^{\left(\bar{\oplus}_{\bar{g}}, \bar{\odot}_{\bar{g}}\right)}\right)(m, f-R M F)=\int_{X}{ }^{\left(\bar{\oplus}_{\bar{g}}, \bar{\odot}_{\bar{g}}\right)} f \bar{\bigodot}_{\bar{g}} d m<+\infty$

The bi- $\left(\bar{\oplus}_{\bar{g}}, \bar{\bigodot}_{\bar{g}}\right)-$ integral in case of the $f_{\bar{g}}-R M F_{\bar{g}}$ with respect to a $\bar{\oplus}_{\bar{g}}-$ measure $m_{\bar{g}}$ [2], [3], [7], [10], [9], [11], [13], [14] will be given below.

Definition 2.1.4: [10] For a real measurable function $f_{\bar{g}}$ (short form $\left.f_{\bar{g}}-R M F_{\bar{g}}\right) f_{\bar{g}}: \bar{g}^{-1}(X) \rightarrow(-\infty,+\infty)$, (in case of the pseudo - operation $\bar{\oplus} \neq \bar{V}$ ), if at least one of the functions $f_{\bar{g}}{ }^{+}, f_{\bar{g}}{ }^{-}$is integrable, then

$\left(B P_{(\bar{g}-T R)}-\int_{\bar{g}^{-1}(X)}^{\left(\bar{\oplus}_{\bar{g}}, \bar{\odot}_{\bar{g}}\right)}\right)\left(m_{\bar{g}}, f_{\bar{g}}-R M F_{\bar{g}}\right)=\left(\int_{\bar{g}^{-1}(X)}^{\left(\bar{\oplus}_{\bar{g}}, \bar{\odot}_{\bar{g}}\right)} f_{\bar{g}}+\bar{\bigodot}_{\bar{g}} d m_{\bar{g}}\right) \bar{\ominus}_{\bar{g}}\left(\int_{\bar{g}^{-1}(X)}^{\left(\bar{\oplus}_{\bar{g}}, \bar{\odot}_{\bar{g}}\right)} f_{\bar{g}}-\bar{\bigodot}_{\bar{g}} d m_{\bar{g}}\right)=$

$=\left(B P_{(\bar{g}-T R)}-\int_{\bar{g}^{-1}(X)}^{\left(\bar{\oplus}_{\bar{g}}, \bar{\odot}_{\bar{g}}\right)}\right)\left(m_{\bar{g}}, f_{\bar{g}}^{+}\right) \bar{\ominus}_{\bar{g}}\left(B P_{(\bar{g}-T R)}-\int_{\bar{g}^{-1}(X)}^{\left(\bar{\oplus}_{\bar{g}}, \bar{\odot}_{\bar{g}}\right)}\right)\left(m_{\bar{g}}, f_{\bar{g}}{ }^{-}\right)$.

A function $f_{\bar{g}}$ is called integrable iff

$-\infty<\left(B P_{(\bar{g}-T R)}-\int_{\bar{g}^{-1}(X)}^{\left(\bar{\oplus}_{\bar{g}}, \bar{\odot}_{\bar{g}}\right)}\right)\left(m_{\bar{g}}, f_{\bar{g}}-R M F_{\bar{g}}\right)=\int_{\bar{g}^{-1}(X)}^{\left(\Phi_{\bar{g}}, \bar{\odot}_{\bar{g}}\right)} f_{\bar{g}} \bar{\bigodot}_{\bar{g}} d m_{\bar{g}}<+\infty$.

\section{The generalization of Bi-Pseudo-Integral and relations with Lebesgue integral}

Proposition 3.1: Let $\mathrm{s}$ be a simple non-negative measurable function (short form $\mathrm{s}$ - SNNMF) defined on $\mathrm{X}$, let $m$ be a $\bigoplus$-measure on $(X, \mathcal{A}),(\bigoplus \neq \mathrm{V})$ and let $g=g_{1, r}$ be a normed generator of the operation $\bigoplus_{g_{a, r}}$. Then the bi-pseudointegral is in the form

$\left(M-\int_{X}\left(\oplus_{a, r} \odot_{g_{a, r}}\right)\right)(m, s-S N N M F)=g_{a, r}{ }^{-1}\left(\frac{1}{a}\right) \bigodot_{g_{a, r}} g_{a, r}{ }^{-1}\left(\int_{X}^{(+, \cdot)}\left(g_{a, r} \circ s\right) \cdot d\left(g_{a, r} \circ m\right)\right)$

where the right-hand side is the Lebesgue integral $\left(L-\int_{X}^{(+, \cdot)}\right)\left(g_{a, r} \circ m, g_{a, r} \circ s-S N N M F\right)$ [3], [7], [10], [11].

Proof. Let $s=\sum_{i=1}^{n} \propto_{i} \cdot \mathbb{I}_{A_{i}}, \propto_{i}>0, A_{i} \in \mathcal{A}$. Then, by definiton 3.1 .1 can get:

$\left(M-\int_{X}\left(\oplus_{g_{a, r}}, \odot_{g_{a, r}}\right)\right)(m, s-S N N M F)=\bigoplus_{i=1}^{\infty} \propto_{i} \cdot m\left(A_{i}\right)$

If we use the equation $x \oplus y=x \bigoplus_{g_{a, r}} y=g_{a, r}{ }^{-1}\left(g_{a, r}(x)+g_{a, r}(y)\right)$ we get:

$\left(M-\int_{X}\left(\oplus_{g_{a, r}} \odot_{g_{a, r}}\right)\right)(m, s-S N N M F)=g_{a, r}{ }^{-1}\left(\sum_{i=1}^{n} g_{a, r}\left(\propto_{i} \cdot m\left(A_{i}\right)\right)\right)=$

$=g_{a, r}{ }^{-1}\left(\sum_{i=1}^{n} a \cdot \propto_{i}^{r} \cdot\left(m\left(A_{i}\right)\right)^{r}\right)=g_{a, r}{ }^{-1}\left(a \cdot \sum_{i=1}^{n} \propto_{i}^{r} \cdot\left(m\left(A_{i}\right)\right)^{r}\right)=$

$=g_{a, r}-1\left(a \cdot \sum_{i=1}^{n} \frac{1}{a} \cdot g_{a, r}\left(\propto_{i}\right) \cdot \frac{1}{a} \cdot g_{a, r}\left(m\left(A_{i}\right)\right)\right)=$

$=g_{a, r}{ }^{-1}\left(\frac{1}{a} \cdot \sum_{i=1}^{n} g_{a, r}\left(\propto_{i}\right) \cdot g_{a, r}\left(m\left(A_{i}\right)\right)\right)=g_{a, r}{ }^{-1}\left(\frac{1}{a} \cdot \sum_{i=1}^{n} g_{a, r}\left(\propto_{i}\right) \cdot g_{a, r}\left(m\left(A_{i}\right)\right)\right)=$ 
$=g_{a, r}{ }^{-1}\left(g_{a, r}\left(g_{a, r}{ }^{-1}\left(\frac{1}{a}\right)\right) \cdot g_{a, r}\left(g_{a, r}{ }^{-1}\left(\sum_{i=1}^{n} g_{a, r}\left(\propto_{i}\right) \cdot g_{a, r}\left(m\left(A_{i}\right)\right)\right)\right)\right)=$

$=g_{a, r}^{-1}\left(\frac{1}{a}\right) \odot_{g_{a, r}}\left(g_{a, r}^{-1}\left(\sum_{i=1}^{n} g_{a, r}\left(\propto_{i}\right) \cdot g_{a, r}\left(m\left(A_{i}\right)\right)\right)\right)=$

$=g_{a, r}^{-1}\left(\frac{1}{a}\right) \odot_{g_{a, r}}\left(g_{a, r}{ }^{-1}\left(\left(L-\int_{X}^{(+;}\right)\left(g_{a, r} \circ m, g_{a, r} \circ s-S N N M F\right)\right)\right)$.

- $\quad\left(M-\int_{X}\left(\oplus_{g_{a, r}, \odot g_{a, r}}\right)\right)(m, s-S N N M F)=\left(M-\int_{X}\left(\oplus_{g_{a, r}}\right)\right)(m, s-S N N M F)=$

$=g_{a, r}{ }^{-1}\left(a \cdot \sum_{i=1}^{n} \alpha_{i}^{r} \cdot\left(m\left(A_{i}\right)\right)^{r}\right)=g_{a, r}{ }^{-1}(a) \odot_{g_{a, r}}\left(g_{a, r}{ }^{-1}\left(\left(L-\int_{X}{ }^{(+;}\right)\left(g_{1, r} \circ m, g_{1, r} \circ S-S N N M F\right)\right)\right)$.

- $\left(\mathrm{M}-\int_{\mathrm{X}}\left(\oplus_{\left.g_{1, r}, \oplus_{g_{1, r}}\right)}\right)(m, s-S N N M F)=\left(\mathrm{M}-\int_{\mathrm{X}}\left(\oplus_{\mathrm{g}_{1, \mathrm{r}^{*}}}\right)\right)(m, s-S N N M F)=\right.$

$=g_{1, r}{ }^{-1}\left(a \oplus_{g_{1, r}}, \odot_{g_{1, r}} \sum_{i=1}^{n} \alpha_{i}^{r} \cdot\left(m\left(A_{i}\right)\right)^{r}\right)=$

$=g_{1, r}^{-1}(a) \odot_{g_{1, r}}\left(g_{1, r}^{-1}\left(\left(L-\int_{X}^{(+,)}\right)\left(g_{1, r} \circ m, g_{1, r} \circ s-S N N M F\right)\right)\right)=$

$=1 \odot_{g_{1, r}}\left(g_{1, r}{ }^{-1}\left(\left(L-\int_{X}^{(+;)}\right)\left(g_{1, r} \circ m, g_{1, r} \circ S-S N N M F\right)\right)\right)=$

$=g_{1, r}^{-1}\left(\left(L-\int_{X}^{(+;)}\right)\left(g_{1, r} \circ m, g_{1, r} \circ S-S N N M F\right)\right)$.

- $\left(\mathrm{M}-\int_{\mathrm{X}}\left(\oplus_{g_{a, r}, \odot g_{a, r}}\right)\right)(m, s-S N N M F)=$

$=\left\{\begin{array}{c}g_{a, r}{ }^{-1}\left(\frac{1}{a}\right) \odot_{g_{a, r}}\left(g_{a, r}{ }^{-1}\left(\left(L-\int_{X}{ }^{(+;}\right)\left(g_{a, r} \circ m, g_{a, r} \circ S-S N N M F\right)\right)\right) \text { for } r \geq 1, a>0 \\ g_{a, r}{ }^{-1}\left(\frac{1}{a}\right) \odot_{g_{a, r}}\left(g_{a, r}{ }^{-1}\left(\left(L-\int_{X}{ }^{(+;}\right)\left(g_{1, r} \circ m, g_{1, r} \circ s-S N N M F\right) \text { for } r \geq 1, a=1\right)\right) \\ g_{1, r}{ }^{-1}\left(\left(L-\int_{X}{ }^{(+;}\right)\left(g_{1, r} \circ m, g_{1, r} \circ s-S N N M F\right)\right) \text { for } r \geq 1, a=1\end{array}\right.$

- $\quad\left(\mathrm{M}-\int_{\mathrm{X}}\left(\oplus_{\left.g_{a, r}, \odot_{g_{a, r}}\right)}\right)(m, s-S N N M F)=\right.$

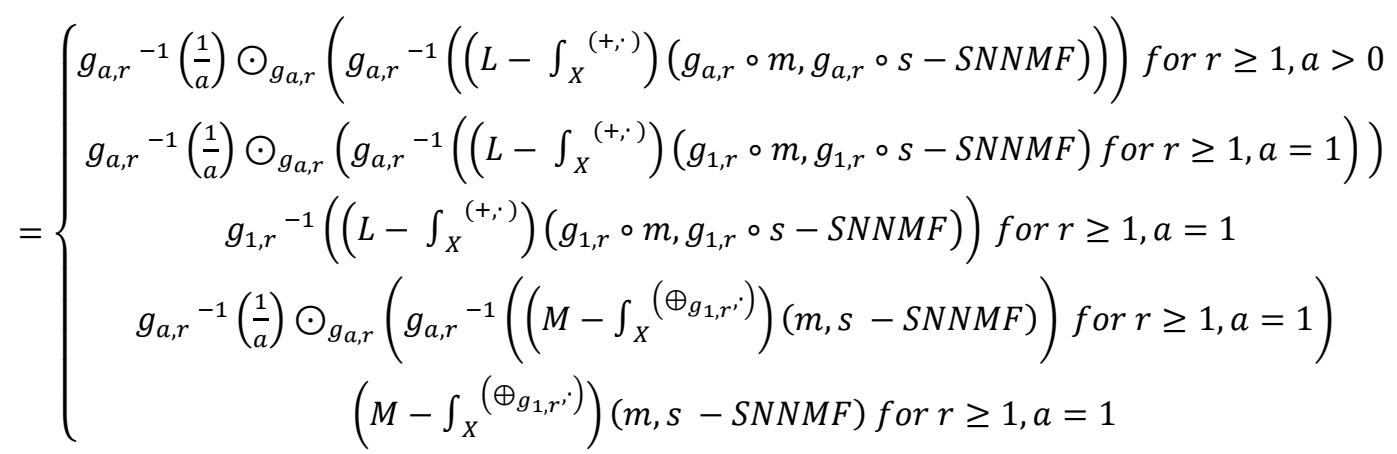

- $\quad\left(\mathrm{M}-\int_{\mathrm{X}}\left(\oplus_{\left.g_{a, r}, \odot_{g_{a, r}}\right)}\right)(m, s-S N N M F)=\right.$ 


$$
=\left\{\begin{array}{c}
a^{-2 / r} \odot_{g_{a, r}}\left(g_{a, r}{ }^{-1}\left(\left(L-\int_{X}{ }^{(+;}\right)\left(g_{a, r} \circ m, g_{a, r} \circ s-S N N M F\right)\right)\right) \text { for } r \geq 1, a>0 \\
\left.a^{-2 / r} \odot_{g_{a, r}}\left(g_{a, r}{ }^{-1}\left(\left(L-\int_{X}{ }^{(+,}\right)\right)\left(g_{1, r} \circ m, g_{1, r} \circ s-S N N M F\right) \text { for } r \geq 1, a=1\right)\right) \\
g_{1, r}{ }^{-1}\left(\left(L-\int_{X}{ }^{(+;}\right)\left(g_{1, r} \circ m, g_{1, r} \circ S-S N N M F\right)\right) \text { for } r \geq 1, a=1 \\
a^{-2 / r} \odot_{g_{a, r}}\left(g_{a, r}{ }^{-1}\left(\left(M-\int_{X}\left(\oplus_{g_{1, r}} \cdot\right)\right)(m, s-S N N M F)\right) \text { for } r \geq 1, a=1\right) \\
\left(M-\int_{X}{ }^{\left(\oplus_{1, r} \cdot\right)}\right)(m, s-S N N M F) \text { for } r \geq 1, a=1
\end{array}\right.
$$

Proposition 3.1.2: [7], [10] Let $(X, \mathcal{A}, m)$ be a $\oplus$ - measure space. The integral of a real measurable $f$ - $R M F$ with respect to a $\bigoplus$-measure $m$, in case $\oplus \neq \mathrm{V}$ (when $\int_{X}{ }^{\left(\bar{\oplus}_{\bar{g}}, \bar{\odot}_{\bar{g}}\right)}$ is defined) is given by:

$\left(M, K-\int_{X}\left(\bar{\oplus}_{\bar{g}}, \bar{\oplus}_{\bar{g}}\right)\right)(m, f-R M F)=\left(M, K-\int_{X}\left(\bar{\oplus}_{\bar{g}_{a, r}, \bar{\odot}_{\bar{g}}}\right)\right)(m, f-R M F)=$

$=\bar{g}_{a, r}{ }^{-1}\left(\bar{g}_{a, r}{ }^{-1}\left(\frac{1}{a}\right) \odot_{g_{a, r}}\left(L-\int_{X}{ }^{(+;}\right)\left(\bar{g}_{a, r} \circ m, \bar{g}_{a, r} \circ f-R M F\right)\right)$

and the integral in the right-hand side $\left(L-\int_{X}{ }^{(+,)}\right)\left(\bar{g}_{a, r} \circ m, \bar{g}_{a, r} \circ f-R M F\right)$ is Lebesgue integral, also $\bar{g}_{a, r} \circ m$ is the Lebesgue measure.

If $\bar{g}$ is the normed generator $\left(\bar{g}=\bar{g}_{1, r}-\right.$ normed generator $)$ hold [3], [7], [10], [11]:

$$
\begin{aligned}
& \left(M, K-\int_{X}{ }^{\left(\bar{\oplus}_{\bar{g}_{1, r}} \bar{\oplus}_{\bar{g}_{1, r}}\right)}\right)(m, f-R M F)=\left(M, K-\int_{X}{ }^{\left(\bar{\oplus}_{\bar{g}_{1, r}}\right)}\right)(m, f-R M F)= \\
& =\int_{X}{ }^{\left(\bar{\oplus}_{\bar{g}_{1, r}}\right)} f \cdot d m=\bar{g}_{1, r}{ }^{-1}\left(\left(L-\int_{X}{ }^{(+;}\right)\left(\bar{g}_{1, r} \circ m, \bar{g}_{1, r} \circ f-R M F\right)\right) .
\end{aligned}
$$

Proof. By using the definition 2.1.4, proposition 3.1 and the additivity of the Lebesgue integral can taken:

$$
\begin{aligned}
& \left(M, K-\int_{X}{ }^{\left(\bar{\oplus}_{\bar{g}}, \bar{\odot}_{\bar{g}}\right)}\right)\left(m, f^{+}\right) \bar{\ominus}_{\bar{g}}\left(M, K-\int_{X}\left(\bar{\oplus}_{\bar{g}}, \bar{\odot}_{\bar{g}}\right)\right)\left(m, f^{-}\right)= \\
& =\int_{X}^{\left(\bar{\oplus}_{\bar{g}}, \bar{\odot}_{\bar{g}}\right)} f^{+} \bar{\odot}_{\bar{g}} d m \bar{\Theta}_{\bar{g}_{a, r}} \int_{X}^{\left(\bar{\oplus}_{\bar{g}}, \bar{\odot}_{\bar{g}}\right)} f^{-} \bar{\bigodot}_{\bar{g}} d m=
\end{aligned}
$$

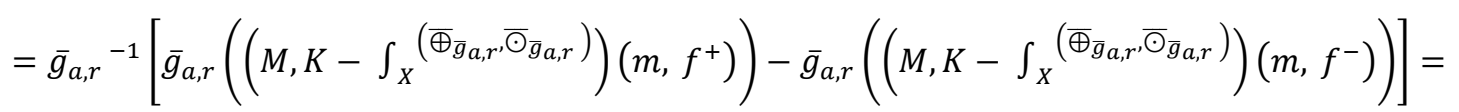

$$
\begin{aligned}
& =\bar{g}_{a, r}{ }^{-1}\left(\bar{g}_{a, r}\left(\int_{X}^{\left(\bar{\oplus}_{\bar{g}_{a, r}} \bar{\odot}_{\bar{g}_{a, r}}\right)} f^{+} \bar{\bigodot}_{\bar{g}_{a, r}} d m\right)-\bar{g}_{a, r}\left(\int_{X}^{\left(\bar{\oplus}_{\bar{g}_{a, r}, \bar{\odot}_{\bar{g}}}\right)} f^{-} \bar{\bigodot}_{\bar{g}_{a, r}} d m\right)\right)= \\
& =\int_{X}^{\left(\bar{\oplus}_{\bar{g}_{a, r}, \bar{\odot}_{\bar{g}} a, r}\right)} f^{+} \bar{\bigodot}_{\bar{g}_{a, r}} d m \bar{\Theta}_{\bar{g}_{a, r}} \int_{X}^{\left(\bar{\oplus}_{\bar{g}}{ }_{a, r}, \bar{\odot}_{\bar{g}_{a, r}}\right)} f^{-} \bar{\odot}_{\bar{g}_{a, r}} d m= \\
& =\bar{g}_{a, r}{ }^{-1}\left\{\bar{g}_{a, r}\left(\left(M, K-\int_{X}\left(\bar{\oplus}_{\left.\bar{g}_{a, r}, \bar{\odot}_{\bar{g}_{a, r}}\right)}\right)\left(m, f^{+}\right)\right)-\bar{g}_{a, r}\left(\left(M, K-\int_{X}\left(\bar{\oplus}_{\bar{g}_{a, r}, \bar{\odot}_{\bar{g}}}\right)\right)\left(m, f^{-}\right)\right)\right\}=\right. \\
& =\bar{g}_{a, r}{ }^{-1}\left\{\bar{g}_{a, r}\left[\bar{g}_{a, r}{ }^{-1}\left(\bar{g}_{a, r}{ }^{-1}\left(\frac{1}{a}\right) \bar{\bigodot}_{\bar{g}_{a, r}} \bar{g}_{a, r}{ }^{-1}\left(\int_{X}{ }^{(+,}\right)\left(\bar{g}_{a, r} \circ f^{+}\right) \cdot d\left(\bar{g}_{a, r} \circ m\right)\right)\right)\right]- \\
& \left.-\bar{g}_{a, r}\left[\bar{g}_{a, r}{ }^{-1}\left(\frac{1}{a}\right) \bar{\odot}_{\bar{g}_{a, r}} \bar{g}_{a, r}{ }^{-1}\left(\bar{g}_{a, r}{ }^{-1}\left(\int_{X}{ }^{(+,)}\left(\bar{g}_{a, r} \circ f^{-}\right) \cdot d\left(\bar{g}_{a, r} \circ m\right)\right)\right)\right]\right\}=
\end{aligned}
$$




$$
\begin{aligned}
& =\bar{g}_{a, r}{ }^{-1}\left\{\left(\bar{g}_{a, r}{ }^{-1}\left(\frac{1}{a}\right) \bar{\bigodot}_{\bar{g}_{a, r}} \bar{g}_{a, r}{ }^{-1}\left(\int_{X}{ }^{(+,}\right)\left(\bar{g}_{a, r} \circ f^{+}\right) \cdot d\left(\bar{g}_{a, r} \circ m\right)\right)\right)- \\
& \left.-\bar{g}_{a, r}\left(\bar{g}_{a, r}{ }^{-1}\left(\frac{1}{a}\right) \bar{\odot}_{\bar{g}_{a, r}} \bar{g}_{a, r}{ }^{-1}\left(\bar{g}_{a, r}{ }^{-1}\left(\int_{X}{ }^{(+,)}\left(\bar{g}_{a, r} \circ f^{-}\right) \cdot d\left(\bar{g}_{a, r} \circ m\right)\right)\right)\right)\right\}= \\
& =\bar{g}_{a, r}{ }^{-1}\left\{\left[\bar{g}_{a, r}{ }^{-1}\left(\frac{1}{a}\right) \odot_{\bar{g}_{a, r}}\left(\left(\int_{X}^{(+,)}\left(\bar{g}_{a, r} \circ f^{+}\right) \cdot d\left(\bar{g}_{a, r} \circ m\right)\right)-\left(\int_{X}^{(+,)}\left(\bar{g}_{a, r} \circ f^{-}\right) \cdot d\left(\bar{g}_{a, r} \circ m\right)\right)\right)\right]\right\}= \\
& =\bar{g}_{a, r}{ }^{-1}\left[\bar{g}_{a, r}{ }^{-1}\left(\frac{1}{a}\right) \odot_{\bar{g}_{a, r}}\left(\int_{X}{ }^{(+,}\left(\bar{g}_{a, r} \circ f\right) \cdot d\left(\bar{g}_{a, r} \circ m\right)\right)\right]= \\
& =\bar{g}_{a, r}{ }^{-1}\left(\bar{g}_{a, r}{ }^{-1}\left(\frac{1}{a}\right) \odot_{g_{a, r}}\left(L-\int_{X}{ }^{(+,}\right)\left(\bar{g}_{a, r} \circ m, \bar{g}_{a, r} \circ f-R M F\right)\right)= \\
& =\int_{X}\left(\bar{\oplus}_{\bar{g}} \bar{g}_{a, r} \bar{\odot}_{\bar{g}_{a, r}}\right) f \bar{\odot}_{\bar{g}_{a, r}} d m=\left(M, K-\int_{X}\left(\bar{\oplus}_{\left.\bar{g}_{a, r}, \bar{\odot}_{\bar{g}_{a, r}}\right)}\right)(m, f-R M F)=\right. \\
& =\left(M, K-\int_{X}{ }^{\left(\bar{\oplus}_{\bar{g}}, \bar{\odot}_{\bar{g}}\right)}\right)(m, f-R M F) .
\end{aligned}
$$

If $\bar{g}$ is the normed generator of $\bar{\oplus},\left.\bar{g}\right|_{[0,+\infty]}=g,\left.\bar{\oplus}\right|_{[0, \infty]}=\bigoplus, g=g_{1, r}-$ normed generator, hold:

$$
\begin{aligned}
& \left(M, K-\int_{X}{ }^{\left(\bar{\oplus}_{\bar{g},} \bar{\odot}_{\bar{g}}\right)}\right)(m, f-R M F)=\left(M, K-\int_{X}{ }^{\left(\bar{\oplus}_{\bar{g}_{1, r}} \bar{\odot}_{\bar{g}_{1, r}}\right)}\right)(m, f-R M F)= \\
& =\left(M, K-\int_{X}{ }^{\left(\bar{\oplus}_{\bar{g}_{1, r}} \cdot\right)}\right)(m, f-R M F)=\int_{X}\left(\bar{\oplus}_{\bar{g}_{1, r}}\right) f \cdot d m= \\
& =\bar{g}_{1, r}{ }^{-1}\left(\bar{g}_{1, r}{ }^{-1}(1) \bigodot_{\bar{g}_{1, r}}\left(L-\int_{X}{ }^{(+;)}\right)\left(\bar{g}_{1, r} \circ m, \bar{g}_{1, r} \circ f-R M F\right)\right)= \\
& =\bar{g}_{1, r}{ }^{-1}\left(1 \odot_{\bar{g}_{1, r}}\left(L-\int_{X}{ }^{(+;}\right)\left(\bar{g}_{1, r} \circ m, \bar{g}_{1, r} \circ f-R M F\right)\right)= \\
& =\left(\left(L-\int_{X}{ }^{(+;)}\right)\left(\bar{g}_{1, r} \circ m, \bar{g}_{1, r} \circ f-R M F\right)\right) .
\end{aligned}
$$

So, all the relations are listed below:

$$
\begin{aligned}
& \left\{\begin{array}{c}
\left(M-\int_{X}{ }^{\left(\oplus_{a, r}, \odot_{g_{a, r}}\right)}\right)(m, s-S N N M F) \text { for } r \geq 1, a>0 \\
g_{a, r}{ }^{-1}\left(\frac{1}{a}\right) \odot_{g_{a, r}}\left(g_{a, r}{ }^{-1}\left(\left(M-\int_{X}\left(\oplus_{g_{1, r}}\right)\right)(m, s-S N N M F)\right) \text { for } r \geq 1, a=1\right)
\end{array}\right.
\end{aligned}
$$

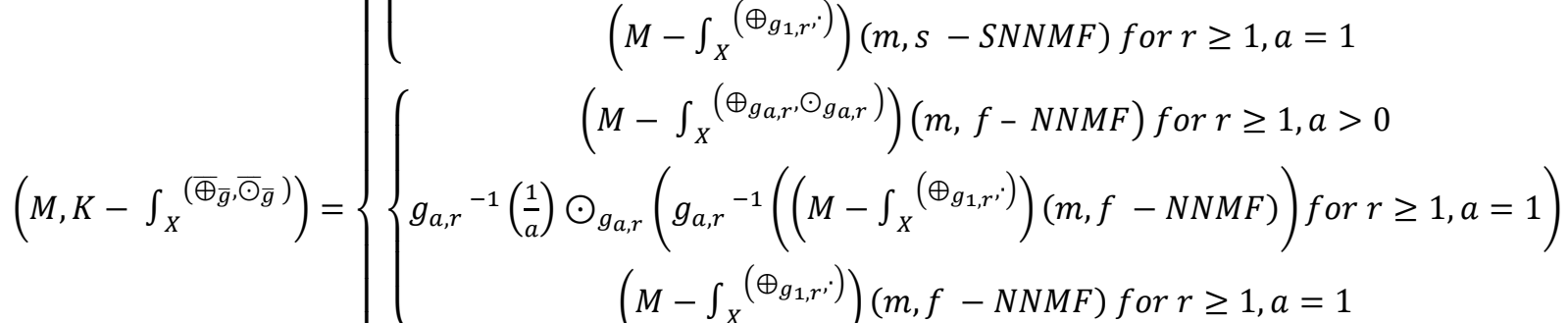

$$
\begin{aligned}
& \left(M-\int_{X}\left(\oplus_{g_{1}, r^{\prime}}\right)\right)(m, f-N N M F) \text { for } r \geq 1, a=1 \\
& \left(M, K-\int_{X}\left(\bar{\oplus}_{\bar{g}_{a, r}, \bar{\odot}_{\bar{g}}}\right)\right)(m, f-R M F) \text { for } r \geq 1, a>0 \\
& \left\{\bar{g}_{a, r}{ }^{-1}\left(\frac{1}{a}\right) \odot_{\bar{g}_{a, r}}\left(\bar{g}_{a, r}{ }^{-1}\left(\left(M-\int_{X}{ }^{\left(\bar{\oplus}_{\bar{g}_{1, r^{\prime}}}\right)}\right)(m, f-R M F)\right) \text { for } r \geq 1, a=1\right)\right. \\
& \left(M, K-\int_{X}\left(\bar{\oplus}_{\bar{g}_{1, r}}\right)\right)(m, f-R M F) \text { for } r \geq 1, a=1
\end{aligned}
$$




\section{The reconstruction of pseudo-additive measure}

Lemma 4.1: Let $(X, \mathcal{A}, m)$ be a $\bar{\oplus}$ - measurable space, $\left(\bar{\oplus}=\bar{\oplus}_{\bar{g}} \neq \bar{V}\right)$. Let $f: X \rightarrow(-\infty, \infty)$ be an integrable function $(f-I F)$. Then the set function $v_{f}(A)=\int_{X}{ }^{\left(\bar{\oplus}_{\bar{g}}, \bar{\odot}_{\bar{g}}\right)} f \bar{\odot}_{\bar{g}} d m=\left(M, K-\int_{X}\left(\bar{\oplus}_{\bar{g}}, \bar{\odot}_{\bar{g}}\right)\right)(m, f)$, for each $A \in \mathcal{A}$, where the bi-pseudo-integral is given by [3], [7], [11] is :

$v_{f}(A)=\left\{\begin{array}{c}\text { a finite } \oplus_{g}-\text { measure on } A \text { if } f-S N N M F \\ \text { a finite } \oplus_{g}-\text { measure on } A \text { if } f-N N M F \\ \text { a finite } \bar{\oplus}_{\bar{g}}-\text { measure on } A \text { if } f-R M F \\ \text { a } \sigma-\bar{\oplus}_{\bar{g}}-\text { measure on } A \text { if } f-R M F \\ \bar{\oplus}_{\bar{g}}=(\cdot)-\text { homogeneous set function on } A \text { if } f-R M F\end{array}\right.$

Proof

1 \& 2. Following Marinová, by Theorem 2 in [11], if $\mathrm{f}-\mathrm{NNMF}$ (or if $\mathrm{f}-\mathrm{SNNMF}$ ), $v_{f}$ is $\oplus_{g}-$ additive and continuous from bellow, where the proof of the continuity is realized in three steps (it is enough by continuity of $g$ and the property of Lebesgue integral)

3. We will consider here the the normed generator ( $\bar{g}=\bar{g}_{1, r}$-normed generator $)$, for $r \geq 1, a=1$. In case of $\left(\bar{\oplus}=\bar{\oplus}_{\bar{g}}=\bar{\oplus}_{\bar{g}_{1, r}} \neq \bar{V}\right)$, the operation $\bar{\oplus}$ is generated by the normed generator $\bar{g}$, and for $A, B \in \mathcal{A}$ with $A \cap B=\phi$ are taken:

$$
\begin{aligned}
& v_{f}(\mathrm{~A}) \bar{\oplus} v_{f}(\mathrm{~B})=v_{f}(B) \bar{\oplus}_{\bar{g}_{1, r}} v_{f}(B)=\bar{\oplus}_{\bar{g}_{1, r}}\left(v_{f}(A), v_{f}(B)\right)= \\
& =\left(M, K-\int_{A}\left(\bar{\oplus}_{\left.\bar{g}_{1, r}, \bar{\odot}_{\bar{g}_{1, r}}\right)}\right)(m, f-R M F) \bar{\oplus}_{\bar{g}_{1, r}}\left(M, K-\int_{B}^{\left(\bar{\oplus}_{\bar{g}_{1, r}, \bar{\odot}_{\bar{g}_{1, r}}}\right)}\right)(m, f-R M F)=\right. \\
& =\int_{A}\left(\bar{\oplus}_{\bar{g}_{1, r}} \bar{\odot}_{\bar{g}_{1, r}}\right) f \bar{\bigodot}_{\bar{g}_{1, r}} d m \bar{\oplus}_{\bar{g}_{1, r}} \int_{B}\left(\bar{\oplus}_{\bar{g}_{1, r}} \bar{\odot}_{\bar{g}_{1, r}}\right) f \bar{\bigodot}_{\bar{g}_{1, r}} d m=\int_{A}\left(\bar{\oplus}_{\bar{g}_{1, r^{\prime}}}\right) f \cdot d m \bar{\oplus}_{\bar{g}_{1, r}} \int_{B}\left(\bar{\oplus}_{\bar{g}_{1, r^{\prime}}}\right) f \cdot d m= \\
& =\bar{g}_{a, r}{ }^{-1}\left[\bar{g}_{1, r}\left(\left(M, K-\int_{A}^{\left(\bar{\oplus}_{\bar{g}_{1, r^{\prime}}}\right)}\right)(m, f)\right)+\bar{g}_{1, r}\left(\left(M, K-\int_{B}^{\left(\bar{\oplus}_{\bar{g}_{1, r^{\prime}}}\right)}\right)(m, f)\right)\right]= \\
& =\bar{g}_{1, r}{ }^{-1}\left(\bar{g}_{1, r}\left(m_{f}(A)\right)+\bar{g}_{1, r}\left(m_{f}(B)\right)\right)=\bar{g}_{1, r}{ }^{-1}\left[\bar{g}_{1, r}\left(\int_{A}^{\left(\bar{\oplus}_{\bar{g}_{1, r}}\right)} f d m\right)+\bar{g}_{1, r}\left(\int_{B}^{\left(\bar{\oplus}_{\bar{g}_{1, r^{\prime}}}\right)} f d m\right)\right]= \\
& =\bar{g}_{1, r}{ }^{-1}\left\{\bar{g}_{1, r}\left[\left(\bar{g}_{1, r}^{-1}\left(\int_{A}^{(+, \cdot)}\left(\bar{g}_{1, r} \circ f\right) d\left(\bar{g}_{1, r} \circ m\right)\right)\right)\right]+\bar{g}_{1, r}\left[\left(\bar{g}_{1, r}{ }^{-1}\left(\int_{B}^{(+, \cdot)}\left(\bar{g}_{1, r} \circ f\right) d\left(\bar{g}_{1, r} \circ m\right)\right)\right)\right]\right\}= \\
& =\bar{g}_{1, r}^{-1}\left\{\int_{A}^{(+;)}\left(\bar{g}_{1, r} \circ f\right) d\left(\bar{g}_{1, r} \circ m\right)+\int_{B}^{(+, \cdot)}\left(\bar{g}_{1, r} \circ f\right) d\left(\bar{g}_{1, r} \circ m\right)\right\}=\bar{g}_{1, r}^{-1}\left[\int_{A \cup B}^{(+, \cdot)}\left(\bar{g}_{1, r} \circ f\right) d\left(\bar{g}_{1, r} \circ m\right)\right]= \\
& =\bar{g}_{1, r}{ }^{-1}\left(\left(L-\int_{X}{ }^{(+, \cdot)}\right)\left(\bar{g}_{1, r} \circ m, \bar{g}_{1, r} \circ f-R M F\right)\right)=\left(M, K-\int_{A \cup B}^{\left(\bar{\oplus}_{\bar{g}_{1, r^{\prime}}}\right)}\right)(m, f-R M F)=\int_{A \cup B}^{\left(\bar{\oplus}_{\bar{g}_{1, r^{*}}}\right)} f d m= \\
& =\int_{A \cup B}^{\left(\bar{\oplus}_{\bar{g}_{1, r}} \bar{\odot}_{\bar{g}_{1, r}}\right)} f d m=\left(M, K-\int_{A \cup B}^{\left(\bar{\oplus}_{\bar{g}_{1, r}, \bar{\oplus}_{\bar{g}_{1, r}}}\right)}\right)(m, f-R M F)=v_{f}(A \cup B) .
\end{aligned}
$$

4. To prove that the set function $v_{f}$ is a finite $\sigma-\bar{\oplus}$ - additive function on $\mathcal{A}$, it is enought to prove its continuity from bellow. Let $A_{n} \in \mathcal{A}, \mathrm{n}=1,2, \ldots$, and let $A_{1} \subset A_{2} \subset \cdots \subset A_{n} \ldots, A_{n}>A$. By the continuity of the generator $\bar{g}=\bar{g}_{1, r}$ and properties of the Lebesgue integral are getting:

$$
\begin{aligned}
& \lim _{n \rightarrow \infty} v_{f}\left(A_{n}\right)=\lim _{n \rightarrow \infty}\left\{\left(M, K-\int_{A_{n}}^{\left(\bar{\oplus}_{\bar{g}_{1, r}} \bar{\odot}_{\bar{g}_{1, r}}\right)}\right)(m, f-R M F)\right\}=\lim _{n \rightarrow \infty} \int_{A_{n}}^{\left(\bar{\oplus}_{\bar{g}_{1, r}} \bar{\odot}_{\bar{g}_{1, r}}\right)} f d m= \\
& =\lim _{n \rightarrow \infty} \bar{g}_{1, r}{ }^{-1}\left(\int_{A_{n}}^{(+,)}\left(\bar{g}_{1, r} \circ f\right) d\left(\bar{g}_{1, r} \circ m\right)\right)=\bar{g}_{1, r}{ }^{-1}\left(\lim _{n \rightarrow \infty}\left(\int_{A_{n}}^{(+;)}\left(\bar{g}_{1, r} \circ f\right) d\left(\bar{g}_{1, r} \circ m\right)\right)\right)=
\end{aligned}
$$




$$
\begin{aligned}
& =\bar{g}_{1, r}{ }^{-1}\left(\int_{A}^{(+, \cdot)}\left(\bar{g}_{1, r} \circ f\right) d\left(\bar{g}_{1, r} \circ m\right)\right)=\bar{g}_{1, r}{ }^{-1}\left(\lim _{n \rightarrow \infty}\left(\int_{A_{n}}^{(+,)}\left(\bar{g}_{1, r} \circ f\right) d\left(\bar{g}_{1, r} \circ m\right)\right)\right)= \\
& =\bar{g}_{1, r}{ }^{-1}\left(\int_{A}^{(+, ;)}\left(\bar{g}_{1, r} \circ f\right) d\left(\bar{g}_{1, r} \circ m\right)\right)=\bar{g}_{1, r}{ }^{-1}\left(\bar{g}_{1, r}\left(\int_{A}^{\left(\bar{\oplus}_{\bar{g}_{1, r}}\right)} f d m\right)\right)= \\
& =\bar{g}_{1, r}{ }^{-1}\left(\bar{g}_{1, r}\left(M, K-\int_{A}^{\left(\bar{\oplus}_{\bar{g}_{1, r}}\right)}\right)(m, f)\right)=\bar{g}_{1, r}{ }^{-1}\left(\bar{g}_{1, r}\left(M, K-\int_{A}^{\left(\bar{\oplus}_{\bar{g}_{1, r}}, \bar{\odot}_{\bar{g}_{1, r}}\right)}\right)(m, f-R M F)\right)= \\
& =\int_{A}^{\left(\bar{\oplus}_{\bar{g}_{1, r}}\right)} f d m=\int_{A}^{\left(\bar{\oplus}_{\bar{g}_{1, r}} \bar{\odot}_{\bar{g}_{1, r}}\right)} f d m=\left(M, K-\int_{A}^{\left(\bar{\oplus}_{\bar{g}_{1, r}, \bar{\odot}_{\bar{g}_{1, r}}}\right)}\right)(m, f-R M F)=v_{f}(A) .
\end{aligned}
$$

By the integrability of $f$, follow finitness of the set function $v_{f}$.

5. The bi-pseudo-integral $\left(M, K-\int_{A}\left(\bar{\oplus}_{\bar{g}} \cdot \cdot\right)\right)$ is a $\bar{\bigodot}_{\bar{g}}=(\cdot)-$ homogeneous functional if $f-R M F$ (in case of $\bar{\oplus}=$ $\left.\bar{\oplus}_{\bar{g}} \neq \overline{\mathrm{V}}\right)$, so

$$
\begin{aligned}
& v_{c \bar{\odot}_{\bar{g}_{1, r}} f}(A)=\left(M, K-\int_{A}\left(\bar{\oplus}_{\left.\bar{g}_{1, r}, \bar{\odot}_{\bar{g}_{1, r}}\right)}\right)\left(m, c \bar{\odot}_{\bar{g}_{1, r}} f-R M F\right)=v_{c \cdot f}(A)=\right. \\
& =\left(M, K-\int_{A}\left(\bar{\oplus}_{\bar{g}_{1, r}}\right)\right)(m, c \cdot f-R M F)=\int_{A}^{\left(\bar{\oplus}_{\bar{g}_{1, r}}\right)} c \cdot f d m=\bar{g}^{-1}\left[\int_{A}^{(+, \cdot)}(\bar{g} \circ c \cdot f) d(\bar{g} \circ m)\right]= \\
& =c \cdot\left\{\bar{g}^{-1}\left[\int_{A}^{(+, \cdot)}(\bar{g} \circ f) d(\bar{g} \circ m)\right]\right\}=c \cdot \int_{A}^{\left(\bar{\oplus}_{\bar{g}_{1, r}}\right)} f d m=c \cdot\left(M, K-\int_{A}\left(\bar{\oplus}_{\bar{g}_{1, r^{\prime}}} \cdot\right)\right)(m, f-R M F)= \\
& =c \cdot v_{f}(A)=c \bar{\odot}_{\bar{g}_{1, r}}\left(M, K-\int_{A}\left(\bar{\oplus}_{\bar{g}_{1, r}} \bar{\odot}_{\bar{g}_{1, r}}\right)\right)(m, f-R M F)=c \bar{\odot}_{\bar{g}_{1, r}} v_{f}(A) .
\end{aligned}
$$

Using relationships that are presented between the bi-pseudo-integrals [10] of different types and classes can be written (in case of $\bar{\oplus}=\bar{\oplus}_{\bar{g}} \neq \bar{V}$ ):

$$
\begin{aligned}
& \left(B P_{(\bar{g}-T R)}-\int_{\bar{g}^{-1}(X)}^{\left(\bar{\oplus}_{\bar{g}_{a, r}} \bar{\odot}_{\bar{g}_{a, r}}\right)}\right)\left(m_{\bar{g}}, f_{\bar{g}}-R M F_{\bar{g}}\right)=\left(M, K-\int_{\bar{g}^{-1}(X)}^{\left(\bar{\oplus}_{\bar{g}_{a, r}, \bar{\odot}_{\bar{g}}}\right)}\right)\left(m_{\bar{g}}, f_{\bar{g}}-R M F_{\bar{g}}\right), \\
& \left(B P_{(\bar{g}-T R)}-\int_{\bar{g}^{-1}(X)}^{\left(\bar{\oplus}_{\bar{g}} \bar{\odot}_{\bar{g}} \bar{g}_{a, r}\right)}\right)\left(m_{\bar{g}}, f_{\bar{g}}-R M F_{\bar{g}}\right)= \\
& =\bar{g}_{a, r}{ }^{-1}\left(a^{-2 / r} \odot_{g_{a, r}}\left(L-\int_{\bar{g}^{-1}(X)}^{(+,)}\right)\left(\bar{g}_{a, r} \circ m_{\bar{g}}, \bar{g}_{a, r} \circ f_{\bar{g}}-R M F_{\bar{g}}\right)\right) .
\end{aligned}
$$

In the same way as the above Lemma 4.1, can be formulated the statements for $v_{f_{\bar{g}}}$ on $\bar{g}^{-1}(A)$ :

$$
v_{f_{\bar{g}}}\left(\bar{g}^{-1}(A)\right)=\left\{\begin{array}{c}
\text { a finite } \oplus_{g}-\text { measure on } g^{-1}(A) \text { if } f_{g}-S N N M F_{g} \\
\text { a finite } \oplus_{g}-\text { measure on } g^{-1}(A) \text { if } f_{g}-N N M F_{g} \\
\text { a finite } \bar{\oplus}_{\bar{g}}-\text { measure on } \bar{g}^{-1}(A) \text { if } f_{\bar{g}}-R M F_{\bar{g}} \\
\text { a } \sigma-\bar{\oplus}_{\bar{g}}-\text { measure on } \bar{g}^{-1}(A) \text { if } f_{\bar{g}}-R M F_{\bar{g}} \\
\bar{\oplus}_{\bar{g}}=(\cdot)-\text { homogeneous set function on } \bar{g}^{-1}(A) \text { if } f_{\bar{g}}-R M F_{\bar{g}}
\end{array}\right.
$$

Proposition 4.2 (The pseudo-linearity of Bi-pseudo-integral):

The extended bi-pseudo-integral for real measurable function [3], [7], [11], (in case of $\bar{\oplus}=\bar{\oplus}_{\bar{g}} \neq \bar{V}$ )

$$
\left(M, K-\int_{X}^{\left(\bar{\oplus}_{\bar{g}}, \bar{\odot}_{\bar{g}}\right)}\right)(m, f-R M F)=\int_{X}^{\left(\bar{\oplus}_{\bar{g}}, \bar{\odot}_{\bar{g}}\right)} f \bar{\odot}_{\bar{g}} d m \text { is: }
$$




$$
\left(M, K-\int_{X}\left(\bar{\oplus}_{\bar{g}}, \bar{\odot}_{\bar{g}}\right)\right)(m, f)=\left\{\begin{array}{c}
\oplus_{g}-\text { functional on } X \text { if } f-S N N M F \\
\oplus_{g}-\text { functional on } X \text { if } f-N N M F \\
\bar{\oplus}_{\bar{g}}-\text { additive functional on } X \text { if } f-R M F \\
\bar{\odot}_{\bar{g}}=(\cdot)-\text { homogeneous functional on } X \text { if } f-R M F
\end{array}\right.
$$

1\& 2. For these cases can be used the same way as [3], [7], and [11].

3. In the conditions that the binary operation $\oplus$ ( pseudo-addition) has been extended $\left(\bar{\oplus}=\bar{\oplus}_{\bar{g}}\right)$ on the interval $[-\infty,+\infty]$, for functions $f_{1}, f_{2}: X \rightarrow[-\infty, \infty]$ can get:

$\left(f_{1} \bar{\oplus}_{\bar{g}} f_{2}\right)(x)=\bar{\oplus}_{\bar{g}}\left(f_{1}(x), f_{2}(x)\right)=\bar{g}^{-1}\left[\left(\bar{g} \circ f_{1}\right)(x)+\left(\bar{g} \circ f_{2}\right)(x)\right]$.

Here, are considered again the the normed generator $\left(\bar{g}=\bar{g}_{1, r}\right.$-normed generator $)$, for $r \geq 1, a=1$ as a general and important generator. In case of $\bar{\oplus}=\bar{\oplus}_{\bar{g}}=\bar{\oplus}_{\bar{g}_{1, r}} \neq \bar{V}$, the operation $\bar{\oplus}$ is generated by the normed generator $\bar{g}$, for all real integrable functions [3], [7], [11] (for which the expressions on both sides make sence) the bi-pseudointegral $\left(M, K-\int^{\left(\bar{\oplus}_{\bar{g}}, \bar{\odot}_{\bar{g}}\right)}\right)$ is $\bar{\oplus}_{\bar{g}}-$ additive functional by:

$$
\begin{aligned}
& \left(M, K-\int_{X}\left(\bar{\oplus}_{\left.\bar{g}_{1, r}, \bar{\odot}_{\bar{g}_{1, r}}\right)}\right)\left(m, f_{1}-R M F\right) \bar{\oplus}_{\bar{g}_{1, r}}\left(M, K-\int_{X}\left(\bar{\oplus}_{\bar{g}_{1, r}, \bar{\odot}_{\bar{g}_{1, r}}}\right)\left(m, f_{2}-R M F\right)=\right.\right. \\
& =\bar{g}_{1, r}{ }^{-1}\left[\bar{g}_{1, r}\left(\left(M, K-\int_{X}\left(\bar{\oplus}_{\bar{g}_{1, r^{\prime}}}\right)\right)\left(m, f_{1}\right)\right)+\bar{g}_{1, r}\left(\left(M, K-\int_{X}\left(\bar{\oplus}_{\bar{g}_{1, r^{\prime}}}\right)\right)\left(m, f_{2}\right)\right)\right]= \\
& =\bar{\oplus}_{\bar{g}_{1, r}}\left(\int_{X}^{\left(\bar{\oplus}_{\bar{g}_{1, r}}\right)} f_{1} d m, \int_{X}^{\left(\bar{\oplus}_{\bar{g}_{1, r^{\prime}}}\right)} f_{2} d m\right)=\int_{X}^{\left(\bar{\oplus}_{\bar{g}_{1, r}}\right)} f_{1} d m \bar{\oplus}_{\bar{g}_{1, r}} \int_{X}^{\left(\bar{\oplus}_{\bar{g}_{1, r^{\prime}}}\right)} f_{2} d m= \\
& =\bar{g}_{1, r}^{-1}\left[\bar{g}\left(\int_{X}^{\left(\bar{\oplus}_{\bar{g}_{1, r^{\prime}}}\right)} f_{1} d m\right)+\bar{g}_{1, r}\left(\int_{X}^{\left(\bar{\oplus}_{\bar{g}_{1, r^{\prime}}}\right)} f_{2} d m\right)\right]= \\
& =\bar{g}_{1, r}{ }^{-1}\left\{\bar{g}_{1, r}\left[\left(\left(M, K-\int_{X}\left(\bar{\oplus}_{\bar{g}_{1, r^{\prime}}}\right)\right)\left(m, f_{1}\right)\right)\right]+\bar{g}_{1, r}\left[\left(\left(M, K-\int_{X}\left(\bar{\oplus}_{\bar{g}_{1, r}}\right)\right)\left(m, f_{2}\right)\right)\right]\right\}= \\
& =\bar{g}_{1, r}{ }^{-1}\left\{\bar{g}_{1, r}\left[\left(\bar{g}_{1, r}^{-1}\left(\int_{X}^{(+;)}\left(\bar{g}_{1, r} \circ f_{1}\right) d\left(\bar{g}_{1, r} \circ m\right)\right)\right)\right]+\bar{g}_{1, r}\left[\left(\bar{g}_{1, r}{ }^{-1}\left(\int_{X}^{(+;)}\left(\bar{g}_{1, r} \circ f_{2}\right) d\left(\bar{g}_{1, r} \circ m\right)\right)\right)\right]\right\}= \\
& =\bar{g}_{1, r}{ }^{-1}\left\{\int_{X}^{(+,)}\left(\bar{g}_{1, r} \circ f_{1}\right) d\left(\bar{g}_{1, r} \circ m\right)+\int_{X}^{(+, \cdot)}\left(\bar{g}_{1, r} \circ f_{2}\right) d\left(\bar{g}_{1, r} \circ m\right)\right\}= \\
& =\bar{g}_{1, r}{ }^{-1}\left[\int_{X}^{(+;)}\left(\bar{g}_{1, r} \circ\left(f_{1} \bar{\oplus}_{\bar{g}_{1, r}} f_{2}\right)\right) d\left(\bar{g}_{1, r} \circ m\right)\right]=\left(L-\int_{X}{ }^{(+;)}\right)\left(\bar{g}_{1, r} \circ m, f_{1} \bar{\oplus}_{\bar{g}_{1, r}} f_{2}-R M F\right)= \\
& =\int_{X}^{\left(\bar{\oplus}^{;}\right)}\left(f_{1} \bar{\oplus}_{\bar{g}_{1, r}} f_{2}\right) d m=\left(M, K-\int_{X}{ }^{\left(\bar{\oplus}_{\bar{g}_{1, r}}, \overline{\bar{g}}_{\bar{g}_{1, r}}\right)}\right)\left(m, f_{1} \bar{\oplus}_{\bar{g}_{1, r}} f_{2}-R M F\right) .
\end{aligned}
$$

4.The bi-pseudo-integral $\left(M, K-\int_{A}{ }^{\left(\bar{\oplus}_{\bar{g}}, \bar{\oplus}_{\bar{g}}\right)}\right)$ is a $\bar{\odot}_{\bar{g}}=(\cdot)$ - homogeneous functional (in case of $\bar{\oplus}=\bar{\oplus}_{\bar{g}}=$ $\left.\bar{\oplus}_{\bar{g}_{1, r}} \neq \overline{\mathrm{V}}\right)$, if $\mathrm{f}-\mathrm{RMF}$, for $c \in(-\infty,+\infty)$ by theorem 1 in [7]:

$$
\begin{aligned}
& \left(M, K-\int_{X}{ }^{\left(\bar{\oplus}_{\bar{g}}, \bar{\odot}_{\bar{g}}\right)}\right)\left(m, c \bar{\odot}_{\bar{g}} f-R M F\right)=\left(M, K-\int_{X}\left(\bar{\oplus}_{\left.\bar{g}_{1, r}, \bar{\odot}_{\bar{g}_{1, r}}\right)}\right)(m, c \cdot f-R M F)=\right. \\
& =\left(M, K-\int_{X}\left(\bar{\oplus}_{\left.\bar{g}_{1, r}, \bar{\odot}_{\bar{g}_{1, r}}\right)}\right)\left(m,(c \cdot f)^{+}\right) \bar{\Theta}_{\bar{g}_{a, r}}\left(M, K-\int_{X}\left(\bar{\oplus}_{\bar{g}_{1, r}, \bar{\odot}_{\bar{g}_{1, r}}}\right)\left(m,(c \cdot f)^{-}\right)=\right.\right. \\
& =\left(\int_{X}^{\left(\bar{\oplus}_{\bar{g}_{1, r}} \bar{\odot}_{\bar{g}_{1, r}}\right)}\left(c \bar{\bigodot}_{\bar{g}_{1, r}} f\right)^{+} \bar{\bigodot}_{\bar{g}_{1, r}} d m\right) \bar{\ominus}_{\bar{g}_{a, r}}\left(\int_{X}^{\left(\bar{\oplus}_{\bar{g}_{1, r}}, \bar{\odot}_{\bar{g}_{1, r}}\right)}\left(c \bar{\odot}_{\bar{g}_{1, r}} f\right)^{-} \bar{\bigodot}_{\bar{g}_{1, r}} d m\right)= \\
& =\left(\int_{X}^{\left(\bar{\oplus}_{\bar{g}}, \bar{\Theta}_{\bar{g}}\right)}(c \cdot f)^{+} \bar{\bigodot}_{\bar{g}_{1, r}} d m\right) \bar{\ominus}_{\bar{g}_{a, r}}\left(\int_{X}^{\left(\bar{\oplus}_{\bar{g}}, \bar{\odot}_{\bar{g}}\right)}(c \cdot f)^{-} \bar{\bigodot}_{\bar{g}_{1, r}} d m\right)=
\end{aligned}
$$




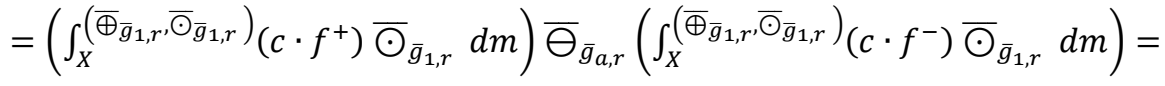

$$
\begin{aligned}
& =c \cdot\left(\int_{X}^{\left(\bar{\oplus}_{\bar{g}_{1, r}} \bar{\odot}_{\bar{g}_{1, r}}\right)} f^{+} \bar{\bigodot}_{\bar{g}_{1, r}} d m \bar{\ominus}_{\bar{g}_{a, r}} \int_{X}^{\left(\bar{\oplus}_{\bar{g}_{1, r}}, \bar{\odot}_{\bar{g}_{1, r}}\right)} f^{-} \bar{\bigodot}_{\bar{g}_{1, r}} d m\right)= \\
& =c \bar{\odot}_{\bar{g}}\left(\int_{X}^{\left(\bar{\oplus}_{\bar{g}_{1, r}} \bar{\odot}_{\bar{g}_{1, r}}\right)} f^{+} \bar{\bigodot}_{\bar{g}_{1, r}} d m \bar{\Theta}_{\bar{g}_{a, r}} \int_{X}^{\left(\bar{\oplus}_{\bar{g}_{1, r}}, \bar{\odot}_{\bar{g}_{1, r}}\right)} f^{-} \bar{\bigodot}_{\bar{g}_{1, r}} d m\right)=
\end{aligned}
$$

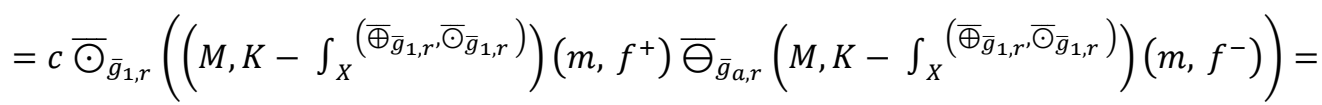

$$
\begin{aligned}
& =c \bar{\bigodot}_{\bar{g}_{1, r}}\left(\left(M, K-\int_{X}{ }^{\left(\bar{\oplus}_{\bar{g}_{1, r}}, \bar{\odot}_{\bar{g}_{1, r}}\right)}\right)(m, f-R M F)\right)=c \bar{\odot}_{\bar{g}}\left(\left(M, K-\int_{X}{ }^{\left(\bar{\oplus}_{\bar{g}}, \bar{\odot}_{\bar{g}}\right)}\right)(m, f-R M F)\right) .
\end{aligned}
$$

Using the definition of bi-pseudo-integrals presented in [10], are summarized (in case of $\bar{\oplus}=\bar{\oplus}_{\bar{g}} \neq \bar{V}$ ):

$$
\left(B P_{(\bar{g}-T R)}-\int_{\bar{g}^{-1}(X)}^{\left(\bar{\oplus}_{\bar{g}}, \bar{\odot}_{\bar{g}}\right)}\right)\left(m_{\bar{g}}, f_{\bar{g}}\right)=\left\{\begin{array}{c}
\oplus_{g}-\text { functional on } g^{-1}(X) \text { if } f_{g}-S N N M F_{g} \\
\oplus_{g}-\text { functional on } g^{-1}(X) \text { if } f_{g}-N N M F_{g} \\
\bar{\oplus}_{\bar{g}}-\text { additive functional on } \bar{g}^{-1}(X) \text { if } f_{\bar{g}}-R M F_{\bar{g}} \\
\bar{\odot}_{\bar{g}}=(\cdot)-\text { homogeneous functional on } \bar{g}^{-1}(X) \text { if } f_{\bar{g}}-R M F_{\bar{g}}
\end{array}\right.
$$

\section{Conclusion}

5.1. The first bi-pseudo-integral $\left(M, K-\int_{\mathrm{X}}\left(\bar{\oplus}_{\bar{g}}, \bar{\odot}_{\bar{g}}\right)\right)(\boldsymbol{m}, \boldsymbol{f})$ in case of the function $\boldsymbol{f}-$ $S N N M F ; N N M F ; R M F$ and relations with Lebesgue integral (depending on the generator $\bar{g}$ ) is summarized as follows

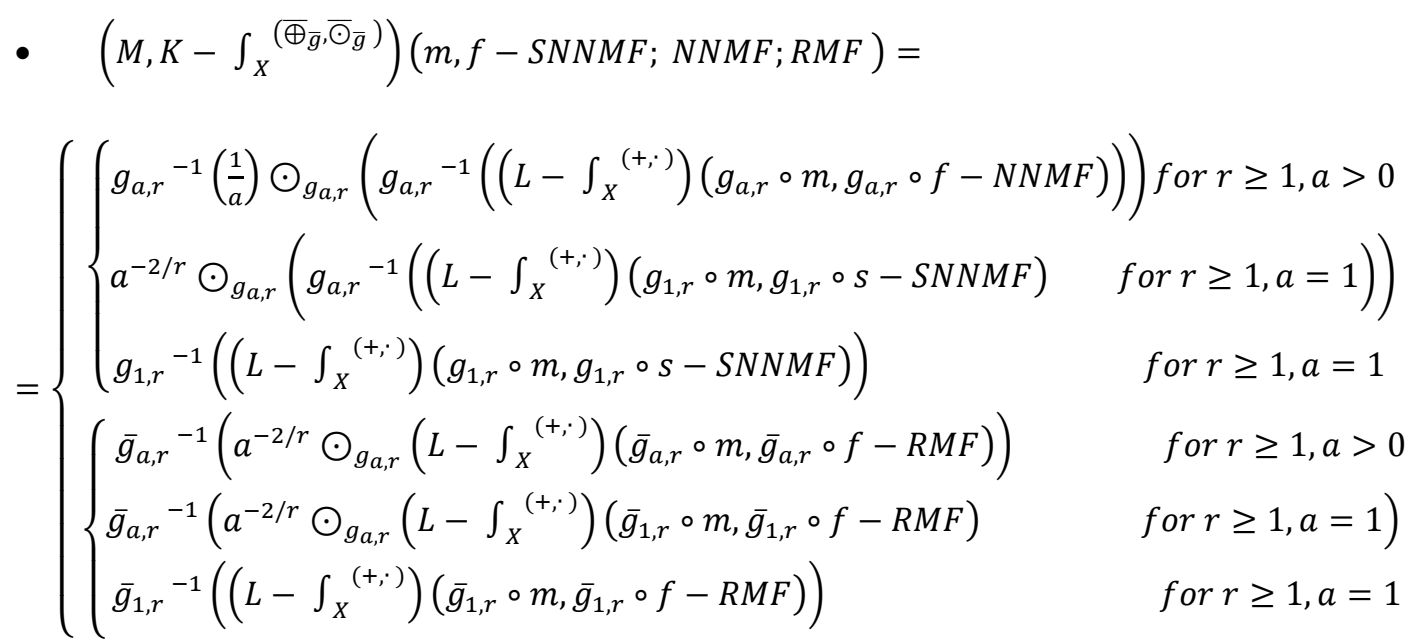

5.2. The first Bi-Pseudo-Integral $\left(M, K-\int_{\mathrm{X}}^{\left(\bar{\oplus}_{\bar{g}}, \bar{\odot}_{\bar{g}}\right)}\right)(m, f)$ in case of the function $f-S N N M F$; NNMF; RMF and the generalizations depending on the generator $\bar{g}$, is summarized as follows

- $\quad\left(\mathrm{M}, \mathrm{K}-\int_{\mathrm{X}}{ }^{\left(\bar{\oplus}_{\bar{g}}, \bar{\odot}_{\bar{g}}\right)}\right)(m, f-S N N M F ; N N M F ; R M F)=$ 


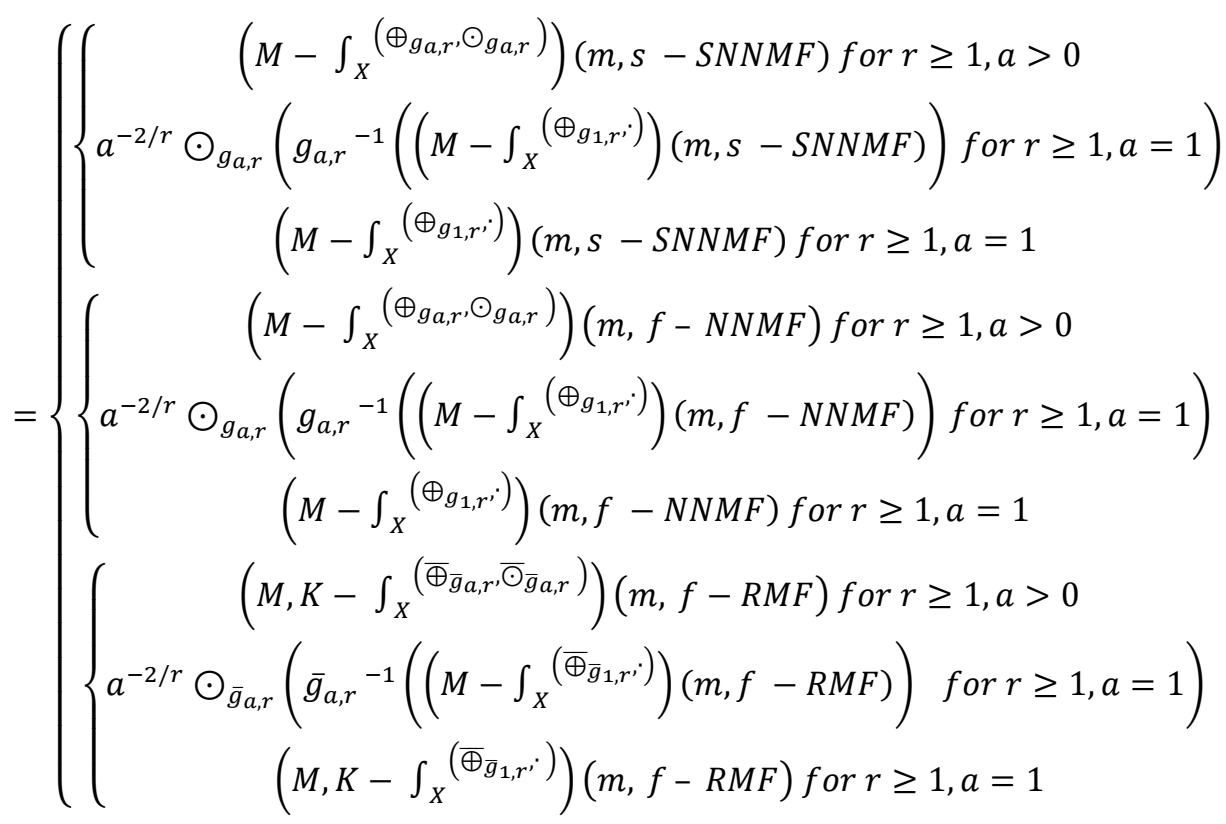

5.3. The first bi-pseudo-integral (with properties of pseudo-linearity) on the reconstruction of pseudo-additive measures when $f-R M F$ :

- $\quad v_{\mathrm{f}}(\mathrm{A})=\left\{\begin{array}{c}\sigma-\bar{\oplus}_{\bar{g}}-\text { measure on } \mathrm{A} \text { if } f-R M F \\ \bar{\oplus}_{\bar{g}}=(\cdot)-\text { homogeneous set function on } \mathrm{A} \text { if } f-R M F\end{array}\right.$

- $\quad\left(M, K-\int_{X}{ }^{\left(\bar{\oplus}_{\bar{g}}, \bar{\odot}_{\bar{g}}\right)}\right)(m, f-R M F)=\left\{\begin{array}{c}\bar{\oplus}_{\bar{g}}-\text { additive functional on } X \\ \bar{\odot}_{\bar{g}}=(\cdot)-\text { homogeneous functional on } X\end{array}\right.$

5.4. The bi-pseudo-integral (with properties of the pseudo-linearity) on the reconstruction of pseudo-additive measures when $f_{\bar{g}}-R M F_{\bar{g}}$ :

- $\quad v_{f_{\bar{g}}}\left(\bar{g}^{-1}(A)\right)=\left\{\begin{array}{c}\sigma-\bar{\oplus}_{\bar{g}}-\text { measure on } \bar{g}^{-1}(\mathrm{~A}) \text { if } f_{\bar{g}}-R M F_{\bar{g}} \\ \bar{\oplus}_{\bar{g}}=(\cdot)-\text { homogeneous set function on } \bar{g}^{-1}(\mathrm{~A}) \text { if } f_{\bar{g}}-R M F_{\bar{g}}\end{array}\right.$

- $\quad\left(B P_{(\bar{g}-T R)}-\int_{\bar{g}^{-1}(X)}^{\left(\bar{\oplus}_{\bar{g}}, \bar{\odot}_{\bar{g}}\right)}\right)\left(m, f_{\bar{g}}-R M F_{g}\right)=\left\{\begin{array}{c}\bar{\oplus}_{\bar{g}}-\text { additive functional on } \bar{g}^{-1}(X) \\ \bar{\odot}_{\bar{g}}=(\cdot)-\text { homogeneous functional on } \bar{g}^{-1}(X) .\end{array}\right.$

\section{Acknowledgements}

First, I would like to give thanks to my professors of NSF, Math. Dep., University of Tirana and to the scientific leader Prof. Dr. A. Tato for the sustention to deepen my knowledge in the science of mathematics and further, for direction and motivation of my scientific research; also the family for giving encouragement and financial support to achieve the scientific research.

\section{References}

[1] J. Acze'1, "Lectures on Functional Equations and their Applications", Academic Press, New York, (1966).

[2] E. Pap, "g-calculus", Univ. u Novom Sadu, Zb. Rad. Prirod.-Mat. Fak. Ser. Mat. 23, 1(1993), pp. $145-156$.

[3] Koles $a^{\prime}$ rova', "A note on the $\bigoplus$-measure based integrals", Tatra Mauntains Math., Publ., 3 (1993), pp .173-182.

[4] R. Mesiar, J. Ryba'rik, "Pseudo-arithmetical operations", Tatra Mauntains Math., Publ., 2 (1993, pp. $185-192$.

[5] N. Ralevic', "Some new properties of g-calculus", Univ. Novom Sadu Zb. Rad. Prirod.-Mat. Fak. Ser. Mat. 24,1 (1994), pp. $139-157$.

[6] Z Riečanova', "About $\sigma$-additive measure and $\sigma$-maxitive measure”, Math. Slovaca, 31 (1982), pp. 389-395.

[7] Kolesárová, "Integration of real functions with respect to a $\bigoplus$-measure", Math.,Slovaca, 46 (1996), No. 1, pp. 41-52.

[8] E. Pap, "Pseudo-additive measures and their applications", in: E. Pap (Ed.), Handbook of Measure Theory, Volume II. Elsevier, Amsterdam, 2002, pp. 1405-1465. http://dx.doi.org/10.1016/B978-044450263-6/50036-1. 
[9] Dh. Valera, I. Dylgjeri, "The special role of the g-functions”, Математички Билтен, ISSN 0351-336X, Vol. 38 (LXIV) No. 1, (2014), Скопје, Македонија, pp. 57-69.

[10] Dh.Valera, "Intercourses with Bi-Pseudo-Integrals based on modified functions and measures by $\bar{g}-$ Transform", Int. Jr. of Mathematical Sciences \& Applications, Mind Reader Publications, ISSN No: 2230-9888, Vol. 5, No. 2, (2015), pp. 401-412.

[11] Marinova", "Integrations with respect to a $\bigoplus$-measure", Math. Slovaca 36, (1986), pp.15-22.

[12] N. Shilkret, "Maxitive measure and integration", Indag. Math., 33 (1971), pp. 109-116 http://dx.doi.org/10.1016/S1385-7258(71)80017-3.

[13] Markova, "Some remark on pseudo-linear algebra", Tatra Mauntains Math., Publ., 6 (1995), pp. 123-130.

[14] J. Ryba'rik," g-FUNCTIONS”, Univ. u Novom Sadu, Zb. Rad. Prirod.-Mat. Fak. Ser. Mat. 25, 1 (1995), pp. 29-38.

[15] M. Sugeno,T. Murofushi, "Pseudo-additive Measure and Integrals", J. Math. Appl.122 (1987), pp. 197-222. http://dx.doi.org/10.1016/0022247x(87)90354-4.

[16] P.Poncet, "Pseudo-multiplication and their properties", arXiv: 1301.0761 [math.RA], 4 Jan 2013. 